\title{
Bioanalysis
}

\section{A standardized kit for automated quantitative assessment of candidate protein biomarkers in human plasma}

\begin{abstract}
Background: An increasingly popular mass spectrometry-based quantitative approach for health-related research in the biomedical field involves the use of stable isotope-labeled standards (SIS) and multiple/selected reaction monitoring (MRM/SRM). To improve inter-laboratory precision and enable more widespread use of this 'absolute' quantitative technique in disease-biomarker assessment studies, methods must be standardized. Results/methodology: Using this MRM-with-SIS-peptide approach, we developed an automated method (encompassing sample preparation, processing and analysis) for quantifying 76 candidate protein markers (spanning $>4$ orders of magnitude in concentration) in neat human plasma. Discussion/conclusion: The assembled biomarker assessment kit - the 'BAK-76' - contains the essential materials (SIS mixes), methods (for acquisition and analysis), and tools (Qualis-SIS software) for performing biomarker discovery or verification studies in a rapid and standardized manner.
\end{abstract}

Considerable effort in quantitative MS has been directed toward the precise and absolute determination of endogenous protein concentrations in human biological samples, most commonly in blood plasma and serum [1]. The motivation for this effort lies in personalized or precision medicine whereby biomarkers (i.e., biological indicators) are sought for enhanced disease monitoring, companion diagnostics, and improved patient outcomes. Quantitation can be accomplished in a number of ways $[2,3]$, with the use of targeted MRMMS and isotopically labeled standard peptides being the most popular implementation within a bottom-up proteomic workflow. In its most basic orientation, an MRM-withSIS-peptide approach can quantify multiplexed panels of plasma proteins of high-tomoderate abundance (i.e., at $\mathrm{mg} / \mathrm{ml}$ to $\mathrm{mid}$ $\mathrm{ng} / \mathrm{ml}$ levels) [4,5], many of which have putative association(s) to disease (e.g., cardiovascular [6,7] and cancer [8-10]). In addition to biomarker analysis, this approach can be extended to address biological queries related to signaling pathways, such as the quantita- tion of epidermal growth factor and estrogen receptor in breast cancer [11] and transcription factors in the innate immune response [12].

In keeping with the strategic direction of the Human Proteome Organization's Plasma Proteome Project [13], standardization harmonization of results between laboratories [14]. This encompasses the pre-analytical (e.g., sample collection and handling, freeze-thaw, and storage stability) and analytical (e.g., sample preparation, processing, and analysis) variables of a proteomic experiment [15-17], which collectively impact the quality of the quantitative results. In an effort to reduce these sources of bias, a compendium of recommendations and standard operating procedures (SOPs) has been assembled (e.g., for standardizing biosample biobanking [18-20]). To minimize the analytical variability, a number of quality control (QC) products have been developed to evaluate the efficacy of individual steps (e.g., indexed retention time (iRT) kit for RT identification and correction [21], QCAL standard for MS assessment [22]) or collective projects are being performed for enhanced
Andrew J Percy ${ }^{1}$, Yassene Mohammed $^{1,2}$, Juncong Yang \& Christoph $\mathrm{H}$ Borchers*,1,3 'University of Victoria - Genome British Columbia Proteomics Centre, Vancouver Island Technology Park, \#3101-4464 Markham St., Victoria, BC V8Z 7X8, Canada

${ }^{2}$ Center for Proteomics \& Metabolomics, Leiden University Medical Center, 2333 ZA, Leiden, The Netherlands ${ }^{3}$ Department of Biochemistry \& Microbiology, University of Victoria, Petch Building Room 207, 3800 Finnerty Rd., Victoria, BC V8P 5C2, Canada *Author for correspondence:

Tel.: +1 2504833221

Fax: +12504833238

christoph@proteincentre.com 


\section{Key terms}

Biomarker: A biological indicator of disease that can be measured routinely - after clinical validation - for diagnostic, prognostic, or predictive purposes. For instance, prostate specific antigen is a US FDA-approved plasma biomarker for prostate cancer; however, its specificity is low with a high false positive rate, which often necessitates unnecessary and invasive prostatic biopsies. This example shows the importance in identifying, verifying, and validating additional markers of disease relevance that can be used in a multimarker test for improved accuracy.

Standardization: A process of defining laboratory practices so that they can be implemented globally for improved data quality and reproducibility.

Quality control: A process that is used to check the efficiency of select or cumulative stages of an experiment (proteomics in this case).

BAK-76: A biomarker assessment kit for quantifying 76 high-to-moderate abundance human plasma proteins that have been associated with various diseases according to studies reported in the literature and online databases.

processes (e.g., DIGESTIF [23], workflow QC kit [24]) within a bottom-up, plasma proteomic experiment. Despite in-roads in biomedical research activities, commercial kits for assessing the biomarker potential of plasma proteins remains rather limited.

Described here is a biomarker assessment kit for the precise, rapid and automated quantitation of 76 high-to-moderate abundance proteins (from 101 interference-free peptide surrogates) in undepleted and non-enriched human plasma. Protein quantitation in the control/patient plasma samples is achieved by linear regression analysis of peptide standard curves. In this strategy, curves are generated from a control sample digest, with the resulting regression information (i.e., slope and y-intercept) being applied to the patient digest analysis for concentration determination. The BAK-76 development involved SIS peptide production, sample prep automation, LC-MS optimization, transition interference evaluation (through ratio analysis in matrix and matrix-free conditions), repeatability/reproducibility assessment, and application testing to patient samples. To enable easy implementation by the proteomics community, key materials (including lyophilized SIS peptide mixtures for control and patient plasma sample analyses) and tools (e.g., SOPs for sample preparation, processing, and analysis; software input files for quantitative analysis with QualisSIS) are provided with the kit. Summarized below are the methods for sample preparation and analysis, the bioanalytical performance of the kit based on our internal evaluations, and the utility of this kit for applying the supplied SIS mixes to control and patient samples in a biomarker-focused study.

\section{Materials \& methods}

Kit material checklist

The BAK-76 provides the following essential materials and tools for the rapid and precise quantitation of 76 disease-related plasma proteins (with possible disease associations from the literature and various online databases noted and referenced in sheet 1 of the Supplementary Excel File):

i. Two lyophilized SIS peptide mixtures for control and patient ( $\mathrm{n}=20$ or 50$)$ human plasma analyses;

ii. Detailed SOPs (includes a troubleshooting guide);

iii. A platform-dependent LC/MRM-MS acquisition method and an acquisition worklist (which contains the sample order and number of replicates, among other processing details) for Agilent's 6490 and 6495 QqQ instruments (extension to other instruments - e.g., Thermo's Q Exactive HF and Sciex's QTRAP 4000 - will be available in the near future);

iv. Platform-dependent Quantitative Analysis method (e.g., Agilent's MassHunter software for the 6490 and 6495 QqQs);

v. Qualis-SIS input CSV files - the peptide template and protein file for control and patient sample analyses for the automated quantitation of processed data via peptide standard curves; and

vi. Guideline values for determining the quantitative accuracy.

These components are distributed to the user by Cambridge Isotope Laboratories as part of the kit.

\section{Internal peptide standards}

Peptide selection was guided by a set of commonlyused sequence-related rules (e.g., the peptide must be unique within the human plasma proteome, devoid of cysteine and methionine residues [25]), but with added emphasis on our previous [4,26] and recent MS/MS results. This directed our selection toward peptides that presented reproducible extracted ion chromatograms (XICs), with the absence of interferences in the MRM ion channels. The 101 peptide surrogates (from 76 proteins) were synthesized (via Fmoc chemistry with ${ }^{13} \mathrm{C} /{ }^{15} \mathrm{~N}$-labels on the C-terminal residue), purified, and characterized, as described previously $[4,26]$. These isotopically labeled standards have the same physicochemical properties as their unlabeled counterparts and therefore experience identical LC-MS behavior (i.e., retention, ionization, and fragmentation - the exception, of course, being in the $\mathrm{m} / \mathrm{z}$ values of the pre- 
cursor ion and any other fragment ions containing the C-terminus). The purity of the characterized targets was found to be $92 \%$, on average (range: $37.8-100 \%$ ), by capillary zone electrophoresis. Peptides were solubilized in $30 \%$ acetonitrile $/ 0.1 \%$ formic acid during the preparation of the SIS peptide mixture. The final mixture was aliquoted, lyophilized, and stored at $-80^{\circ} \mathrm{C}$ until immediately prior to use, at which time they were rehydrated in dilute formic acid (at $0.1 \%$ ) prior to their addition to the digested samples. No peptide solubility issues have been observed during reconstitution.

\section{Automated sample preparation}

To facilitate implementation and improve method reproducibility, our manual preparation methods for human plasma (e.g., [4,24]) have been modified to allow automation on a Tecan Freedom EVO 150. This involved automating all of the liquid handling steps associated with plasma protein preparation (which includes denaturation, disulfide bond reduction, and cysteine alkylation), trypsinization, SIS peptide addition, and solid phase extraction (SPE) on a 96-well microplate (Axygen, CA, USA). To accommodate this, the Tecan was configured with an 8-channel pipet head, block modules for 96-well plates (we use 1.1-ml wells), a shaker platform, a reagent trough and screw cap tube station (we use $100 \mathrm{ml}$ troughs and $10 \mathrm{ml}$ tubes), a SPE device with vacuum manifold, and a robotic arm fitted for the use of liquid handling $(\mathrm{LiHa})$ disposable pipette tips (200 $\mu \mathrm{l}$ or $1 \mathrm{ml}$, without filters). Before operating the Tecan workstation, solutions of tenfold diluted plasma (collected initially in sterile tubes containing $\mathrm{K}_{2}$.EDTA anticoagulant from 30 healthy donors and 20 randomly selected de-identified patients, then supplied as a pooled control sample and 20 individual patient samples by Bioreclamation, NY, USA), $25 \mathrm{mM}$ ammonium bicarbonate, $10 \%$ sodium deoxycholate, and $50 \mathrm{mM}$ tris-(2-carboxyethyl)phosphine were prepared in a trough or tube for protein denaturation and disulfide bond reduction. Solutions of $100 \mathrm{mM}$ iodoacetamide, $100 \mathrm{mM}$ dithiothreitol, and $2 \mathrm{mg} / \mathrm{ml}$ Worthington trypsin (in $25 \mathrm{mM}$ ammonium bicarbonate) were prepared immediately prior to their sequential addition.

In terms of the incubations, denaturation and reduction were performed simultaneously at $60^{\circ} \mathrm{C}$ for $30 \mathrm{~min}$, alkylation and quenching were sequentially performed for $30 \mathrm{~min}$ at $37^{\circ} \mathrm{C}$, while proteolysis (using a 10:1 substrate:enzyme ratio) was allowed to proceed for $16 \mathrm{~h}$ at $37^{\circ} \mathrm{C}$. At the time of proteolysis, each sample well contains $350 \mu$ li.e., $166.5 \mu \mathrm{l}$ of $25 \mathrm{mM}$ ammonium bicarbonate, $30 \mu \mathrm{l}$ of sodium deoxycholate, $50 \mu \mathrm{l}$ of diluted plasma ( $10 \mu \mathrm{l}$ initial), $28 \mu \mathrm{l}$ of $50 \mathrm{mM}$ tris-(2-carboxyethyl)phosphine, $29 \mu \mathrm{l}$ of $100 \mathrm{mM}$ iodoacetamide, $29 \mu \mathrm{l}$ of $100 \mathrm{mM}$ dithiothreitol, and $17.5 \mu \mathrm{l}$ of $2 \mathrm{mg} / \mathrm{ml}$ trypsin). Note that this procedure is repeated in six separate wells with the pooled plasma control for the generation of 6-point standard curves. The curves contain a constant amount of the endogenous peptides and a variable amount of the concentration-balanced isotopically labeled peptide mixture (spiked in post-digestion, as described below).

After overnight digestion, an acidified concentrationbalanced SIS peptide mixture $(51 \mu \mathrm{l}$ per well, at a constant concentration of $25 \mathrm{fmol} / \mu \mathrm{l}$ in the patient analysis or $250-0.5 \mathrm{fmol} / \mu \mathrm{l}$ in the control analysis) and $2.7 \%$ formic acid ( $40 \mu \mathrm{l}$ per well) were combined with an aliquot of each digest $(126 \mu \mathrm{l})$ in a separate 96 -well plate. Further to the SIS mix, the SIS peptides function as internal standards in the patient analysis, where they help normalize all post-digestion steps (includes SPE and LC/MRM-MS) toward the precise and relative quantitation of 76 target proteins. The SIS peptides have been 'concentration-balanced' to match the concentrations of their corresponding endogenous natural (NAT) peptides in a control plasma digest, returning relative response (i.e., NAT/SIS peak area) ratios approximating $1: 1$. The use of a concentration-balanced mixture is preferred to an equimolar mixture as it has been demonstrated to improve the precision of the assay [27]. In the control analysis, the most concentrated SIS-peptide mixture (at $250 \mathrm{fmol} / \mu \mathrm{l}$ ) is serially diluted (at ratios of 1:2:5:2:5:5 from the most concentrated level F) and spiked into the plasma digest to give 6-point standard curves spanning a 500-fold concentration range. In our quantitative approach, the NAT peptides function as internal standards in the control analysis.

Returning to the sample preparation, the $\mathrm{pH}$ reduction due to the formic acid causes the deoxycholate surfactant to precipitate, which can then be pelleted by centrifugation $\left(3000 \mathrm{rpm}\right.$ for $5 \mathrm{~min}$ at $22^{\circ} \mathrm{C}$; rotor S2096; Allegra X-22R; Beckman Coulter, MO, USA) and the resulting supernatant $(144 \mu \mathrm{l})$ desalted by SPE (Oasis $30 \mu \mathrm{m}$ HLB microelution plate). After elution with $50 \%$ acetonitrile $/ 0.1 \%$ formic acid $(75 \mu \mathrm{l})$ and lyophilization, each well was rehydrated with $83 \mu \mathrm{l}$ of $0.1 \%$ formic acid for LC/MRM-MS (final concentration: $1 \mu \mathrm{g} / \mu \mathrm{l})$.

\section{Sample processing by UHPLC/MRM-MS}

The LC-MS platform for the BAK-76 evaluations consisted of a reversed-Phase UHPLC column (2.1 $\times 150 \mathrm{~mm}, 1.8 \mu \mathrm{m}$ particles; part no. 959759-902; Agilent Technologies, CA, USA) interfaced to a triple quadrupole (QqQ) mass spectrometer (Agilent's 6490 or 6495) via Agilent's Jet Stream source. The column was maintained in a thermostatted compartment (at $50^{\circ} \mathrm{C}$ ) within a 1290 Infinity UHPLC system (Agilent 
Technologies). Peptide mixtures $(15-\mu \mathrm{l}$ injections from a 96-well plate housed in a $4{ }^{\circ} \mathrm{C}$ autosampler) were separated at a flow rate of $0.4 \mathrm{ml} / \mathrm{min}$ using the following ACN gradient (time, eluent $\% B$ ): $0,1.5 ; 1.5,6.3 ; 15.5$, $13.3 ; 17.5,16.6 ; 23,20 ; 25.5,25.5 ; 26.5,40.4 ; 27,81$; 29, 81; and 30, 1.5. The mobile phase compositions were $0.1 \%$ formic acid in either $100 \%$ water (for $\mathrm{A}$ ) or $100 \%$ acetonitrile (for B). Each run was followed by an injection of $0.1 \%$ formic acid at the starting elution conditions of $1.5 \% \mathrm{~B}$ for a 4 min column equilibration.

The LC operating details, along with all pertinent MS parameters (e.g., capillary voltage of $3.5 \mathrm{kV}$, nozzle voltage of $300 \mathrm{~V}$, sheath gas at $11 \mathrm{l} / \mathrm{min}$ and $250^{\circ} \mathrm{C}$, unit mass resolution) for optimal peptide ionization, fragmentation, and detection are provided in the kit's 'platform-dependent LC/MRM-MS acquisition method' (see Supplementary Table 1 for the specific acquisition parameters contained in the method). For the Agilent $6495 \mathrm{QqQ}$, manual adjustment of the high energy dynode multiplier to $-20 \mathrm{kV}$ in the acquisition tuning file is needed for improved ion detection efficiency and signal-to-noise ratios [Miller CA, Pers. Comm. (2014)]. The final dynamic MRM method consists of 606 MRM transitions, with 60 being the maximum number that are concurrently monitored during a given $700 \mathrm{~ms}$ cycle. This provides minimum dwell times of $10.76 \mathrm{~ms}$, which is facilitated by narrow MRM detection windows of $1 \mathrm{~min}$ (i.e., $\pm 0.5 \mathrm{~min}$ from scheduled retention time).

In terms of the run order and number of replicates, the 6 concentration levels used to generate the standard curve are processed from lowest (i.e., standard A) to highest (i.e., standard F) in triplicate, with two blanks inserted between each block of standard measurements, as follows: standards A-F replicate 1, blank 01, blank 02, standards A-F replicate 2, blank 03, etc. After the curve control samples, single injections of each patient sample are made. These details are indicated in the kits 'platform-dependent LC/MRM-MS acquisition worklist.'

\section{Automated data analysis}

MRM data are viewed with MassHunter Quantitative Analysis software (version B.07.00, Agilent) and quantified with Qualis-SIS (University of Victoria - Genome BC Proteomics Centre; available at bioinformatics.proteincentre.com:3838/qualis-sis/) by standard curves and regression analysis (described mathematically in [4] and computationally in [28]). The provided 'platformdependent MassHunter Analysis method' contains all of the necessary parameters (e.g., peak area threshold, peak selection criteria) and has the quantifier/qualifier transitions pre-selected. The Qualis-SIS input files required for the analyses of the control and patient samples are provided as a template in the peptide file (where the responses for the quantifier transitions have been left blank), and as a fixed file for the protein (containing, e.g., SIS peptide concentrations and protein molecular weights). The default parameters (e.g., $1 / \mathrm{x}^{2}$ regression weighting, $<20 \%$ deviation in a level's precision/accuracy, inclusion of a minimum of 3 consecutive concentration levels) in Qualis-SIS are to be used for the automated generation of standard curves and the subsequent quantitation of patient samples. The curves are generated if, and only if, these qualification parameters are adhered to, with the resulting concentrations being acceptable only if they lie within the assay's range of linearity (i.e., between lower and upper limits of quantitation). All performance metrics of the standard curves (e.g., concentration, limits of quantitation, dynamic range, coefficient of determination, precision in relative response, retention time, and peak width) are provided in downloadable Excel files from the Qualis-SIS 'File’ page.

\section{Results \& discussion}

\section{Kit panel origination}

Through a combination of linear regression and single point measurement (SPM), we have previously quantified large panels of plasma proteins with simple sample preparation and unidimensional LC/MRM-MS sample processing $[4,26]$. From these panels, a collection of higher-abundance proteins were selected for our first BAK, with future plasma-based assessment kits to be focused on broader panels and proteins of lower abundance. Extensive preliminary evaluations involved intra- and inter-assay precision testing with manual and automated sample preparation performed in process replicate $(n=5)$ by technicians of varying skill levels. Although these results will be presented separately, the essential outcome was the need for standardization and the reproducibly quantified panel of 76 plasma proteins. These were inferred from 101 interferencefree peptides and yielded an inter-assay precision of $7.8 \% \mathrm{CV}$ in average concentration over process replicate (see Figure 1 for the XIC traces and Figure 2 for the quantitative reproducibility).

\section{Robust automated sample preparation \& processing}

After conducting a series of preliminary optimizations (relating to the LC gradient and the transition acquisition parameters for MRM on the 6495) and evaluations (e.g., robotic system setup, SIS mix testing in buffer and digest for interference screening), a number of validation tests and pilot studies were performed to demonstrate the quantitative reproducibility and the kit utility. To assess the reproducibility of the Tecan 


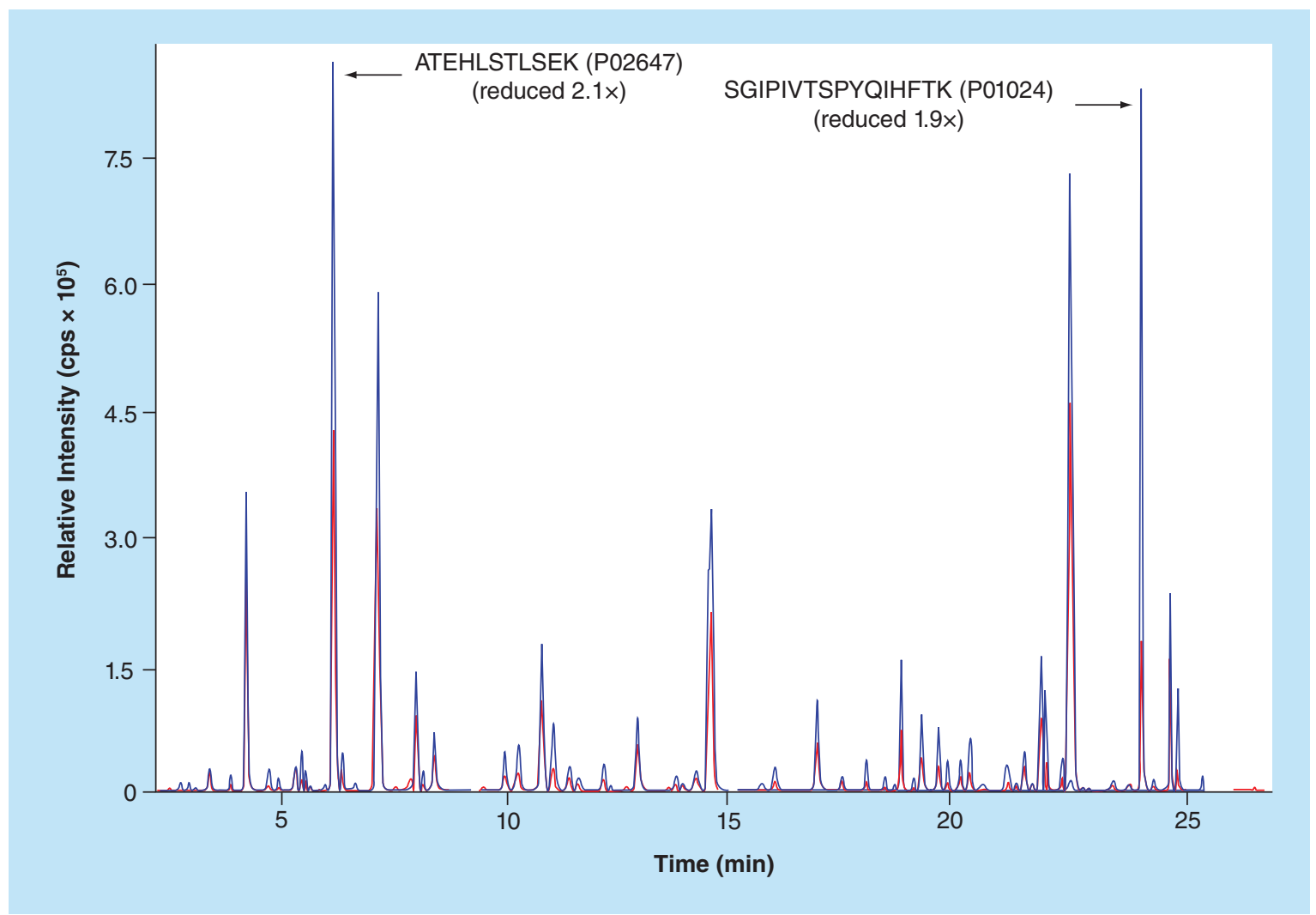

Figure 1. Representative XIC trace from the automated analysis of the control plasma sample by LC/MRM-MS on an Agilent 6495 QqQ mass spectrometer. For clarity, only the quantifier transitions of the 101 peptides are shown, with the SIS peptides marked in red and their corresponding natural forms in blue. The retention times and transition details are provided in Supplementary Table 1.

robotic system in combination with the LC-MS platform, 50 identical plasma samples were independently prepared from the same batch of starting materials and processed by LC/MRM-MS on a 96-well plate immediately following solid phase extraction. The results revealed excellent inter-assay reproducibility of retention time $(0.09 \% \mathrm{CV}$, on average), peak width $(1.8 \% \mathrm{CV}$, on average), and signal $(9.4 \% \mathrm{CV}$, on average, for the relative response of 101 peptides) over the two days of sample measurements (total instrument time was $\sim 30 \mathrm{~h}$; see Supplementary Table 2 for the variability results). This level of performance is in general accord with that found previously using a similar workflow and analytical platform, but with different standard peptide mixtures $[4,24,29,30]$. This provides confidence in the pipetting efficiency and the robustness of the automated platform.

\section{Quantitative reproducibility}

\section{\& quantifier/qualifier assignments}

The concentrations of the target peptides spanned $>4$ orders of magnitude - from average values of $8 \mathrm{mg} / \mathrm{ml}$ for ceruloplasmin (CP, P00450) to $448 \mathrm{ng} / \mathrm{ml}$ for complement Clq subcomponent subunit A (C1QA, P02745) - across the 50 control sample measurements.
These SPM-derived values are in general agreement with those reported previously by us $[4,26]$ and others $[17,24,31-39]$ where different analytical and pre-analytical variables were used to quantify proteins in control plasma samples. For example, an $8.3 \%$ variability in the concentration of vitamin K-dependent protein S (PROS1, P07225; a candidate marker for cardiovascular disease [39]), was found between the average value determined here $(23 \mu \mathrm{g} / \mathrm{ml})$, our previous in-house MRM measurements [26,30], and values obtained from other LC-MS/MS [17] and ligandbinding assays (e.g., ELISA [39]). Of greater importance, however, is the correlation between the SPM and the standard curve results derived here, since these were generated from similar materials processed under an identical LC-MS/MS workflow. Using our stringent calibration-curve criteria, defined in the 'Automated Data Analysis' portion of the experimental section, qualification was achieved for all 101 peptides (see Supplementary Table 3 for the standard curve-extracted metrics), with the resulting concentrations providing excellent correlation with the SPM-derived values (i.e., a coefficient of determination $\left(\mathrm{R}^{2}\right)$ of 0.999 from a plot of the raw values). In these concentration determinations, the quantifier transition of a given peptide was assigned 


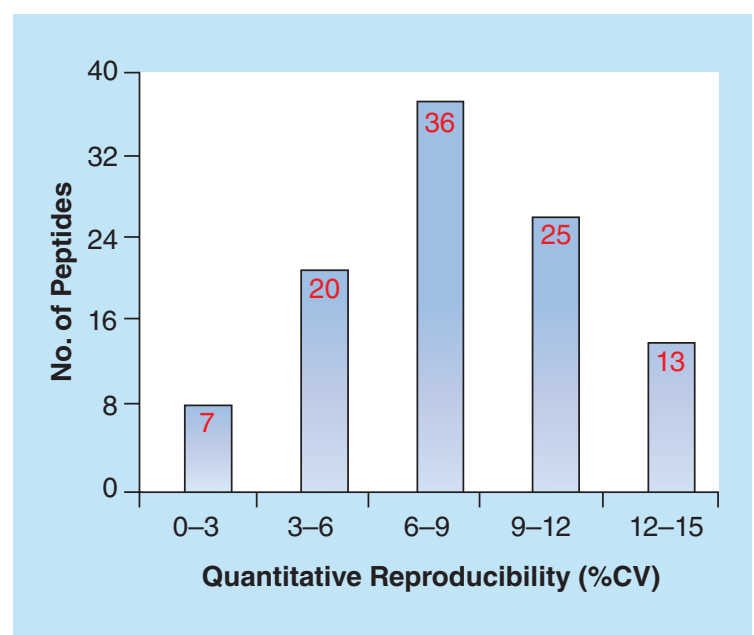

Figure 2. Variability in determined protein concentrations as determined from interassay reproducibility testing. Concentrations were measured in process replicates $(n=5)$ and were calculated using Qualis-SIS based on peptide standard curves with $<15 \%$ CV.

to the transition that provided the lowest limit of quantitation (LOQ), while the remaining two transitions served as retention time qualifiers for peak verification. The use of a single transition is justified for peptide quantitation since the MRM approach offers five dimensions of analyte specificity (i.e., precursor and product ion $\mathrm{m} / \mathrm{z}$ values, retention time, peak shape, and relative response) and transitions are rigorously assessed for chemical interferences (by examining ion-transition ratios) during assay development. In cases where the detection sensitivities of the SIS and NAT peptides both decrease in the plasma samples as a result of matrix effects, it is important to note that the relative responses (i.e., NAT/SIS peak area ratios), and therefore the determined concentrations, remain unchanged. To summarize, it is the combination of careful interference screening and the use of relative responses (made possible by the use of well-characterized SIS peptides) that helps overcome the matrix effects.

The target panel of the BAK- 76 contains 25 proteins that are represented by two quantitative peptide surrogates. For simplicity, the reported protein concentration can be based on the peptide that yields the highest concentration (referred to as the quantifier peptide), since it is assumed to have been digested and liberated with the greatest efficiency. Figure 3 illustrates the concentration range determined for the 76 plasma proteins in the control sample (as derived by the quantifier peptides), with an inset showing the distribution of their linear dynamic ranges (defined as the ULOQ divided by the LLOQ) obtained from each peptide's standard curve. Also shown as an inset is a table highlighting the agreement between the concentrations derived here and those reported in the literature. Overall, the plasma concentrations of all 101 peptides were found to lie within their assay's linear dynamic range, being an average of 39.7-fold higher than their LLOQs and one-sixth of their ULOQs.

Although quantitation is typically based on the quantifier peptide, monitoring multiple peptides for a given protein is still useful since their concentrations can be compared, on a per-protein basis, for interference screening of the experimental sample results $[4,41]$. When used in this way, deviations from linearity indicate the presence of an interference in a particular sample that can later be verified or refuted through inspection of the peptide XIC traces (see Figure 4 in reference [4] for an example). For the plasma sample measured here, the endogenous protein concentrations determined from different peptides representing the same protein were predominantly within two-fold (see Figure 5 for the fold changes). Interestingly, peptides from fibulin-1 (FBNL1, P23142; giving concentrations of 12.9 and $12.5 \mu \mathrm{g} / \mathrm{ml}$ ) and protein AMBP (AMBP, P02760; giving concentrations of 20.6 and $21.7 \mu \mathrm{g} / \mathrm{ml}$ ) showed the best correlation. For the 7 proteins that presented fold-changes of $>2$, increasing the substrate:enzyme ratio (from 10:1 to 5:1) failed to improve the correlation, as revealed by a paired $t$ test evaluation. This suggests that the cause is structurebased and relates to reduced denaturation/reduction efficiency, rather than digestion efficiency.

\section{Practicality of the BAK-76}

To demonstrate the application of the BAK-76 to authentic samples, the control sample was again processed together with a set of 20 randomly selected, patient plasma samples. After confirming system QC with the daily LC-MS platform performance kit, the standard curves from the control sample were found to deliver excellent linearity (average $\mathrm{R}^{2}$ of 0.985 ) and a broad dynamic range (average of 165), with stable chromatographic characteristics (average intra-assay CVs of $0.09 \%$ for retention time and $10.9 \%$ for signal) obtained for the NAT signals across the replicates of all 6 concentration levels. If imprecision is observed (i.e., $>20 \%$ deviation in the average precision of replicate analyses of a single concentration level; revealed in the comprehensive results export of Qualis-SIS) and Qualis-SIS was used to guide peak acceptability, then the user should inspect the XICs in the response generation software (be it MassHunter Quantitative Analysis or Skyline [42,43]) before re-analysis with QualisSIS. In our experience, if the preparation protocol and the recommended detection conditions are adhered to, inspection of the SIS signals in the control sample analysis should be only required for the 2 lower concentration levels of the standard curve. For the NAT signals, the responses of their quantifier transitions should be 


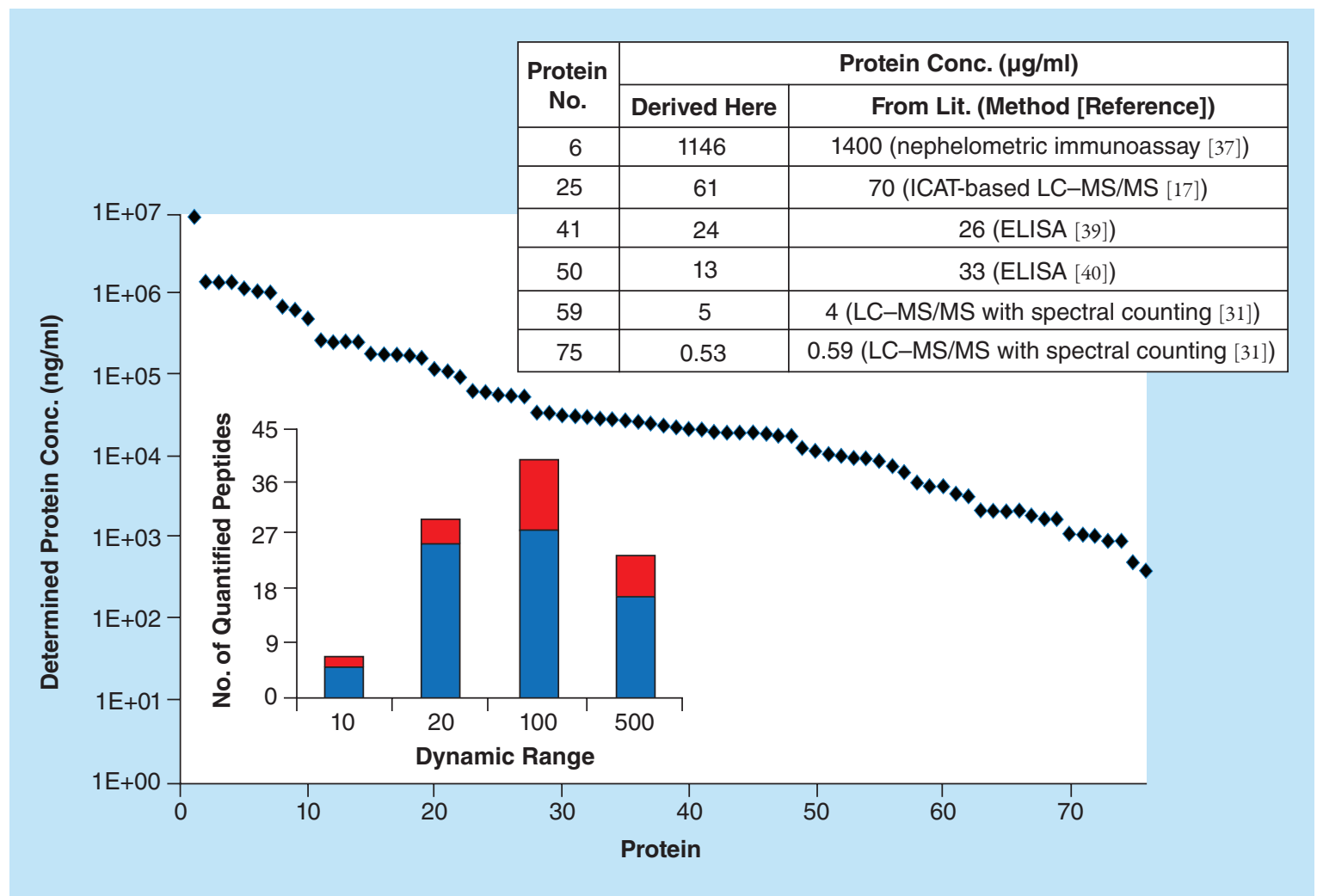

Figure 3. Concentration range for the quantifier peptides of the plasma proteins in the BAK-76 determined by an MRM-with-SIS-peptide approach. The concentrations were obtained from peptide standard curves that contained a constant amount of NAT and a 500-fold range of SIS. The histogram in the inset shows the linear dynamic range obtained from quantifying the 101 target peptides in the control sample via standard curves, with blue denoting the quantifier peptides and red denoting the qualifier peptides. The table inset compares the concentrations determined here with those reported in the literature from other quantitative techniques - protein 6 [37], protein 25 [17], protein 41 [39], protein 50 [40], and proteins 59 and 75 [31]. The protein numbers, concentrations and other assay metrics are provided in Supplementary Table 3.

consistently strong, providing areas of approximately $3.8 \mathrm{E}+05$, on average. In the control plasma digest measured here, peptide ATEHLSTLSEK from apolipoprotein A-1 (APOA1, P02647) yielded the highest signal (at 9.0E+06) and peptide ESHVTLASPEETR from vasorin (VASN, Q6EMK4) yielded the lowest (at $1.2 \mathrm{E}+03)$. The same general approach for peak confirmation/inspection applies to patient samples that are run on multiple 96-well plates.

In terms of the patient results, the sample concentrations revealed up-/down-regulation that were protein- and sample-dependent (see the raw and relative concentrations tabulated in sheet 2 of the Supplementary Excel file). For apolipoprotein(a) (LPA, P08519), for example, up-regulation was observed for 2 subjects (samples 1 and 20; with average relative concentrations with respect to the control of 4.5) and down-regulation for 7 subjects (samples 4, 5, 8, 9, 15, 18 , and 19; with an average relative concentration with respect to the control of 3.3). In contrast, up-regulation of CD5 antigen-like protein (CD5L, O43866) was observed for 2 subjects (samples 4 and 6; average relative concentration with respect to the control of 2.1), while down-regulation was not observed in the 20 samples investigated. Regarding the determined concentrations for the patient samples, these were largely found to lie within the reference ranges reported by noted clinical laboratories (see Supplementary Excel Table 2 in [35]), which validates this quantitative approach and the resulting values. This correlation is illustrated in Figure 6 for two high-abundance proteins (apolipoprotein A-I [APOA1, P02647] and apolipoprotein B-100 [APOB, P04114]) and two moderate-abundance proteins ( $\beta-2-$ microglobulin [B2M, P61769] and cystatin$\mathrm{C}$ [CST3, P01034]). Interestingly, similar concentration fold-changes were obtained for the 25 proteins quantified by two peptides in the control and patient analysis (e.g., 1.23 vs an average of 1.18 for fibrinogen alpha chain [FIBA, P02671], and 7.53 vs an average of 7.23 for clusterin [CLUS, P10909]). This affirms the reproducibility of the proteolysis step in the targeted quantitation of these proteins. 


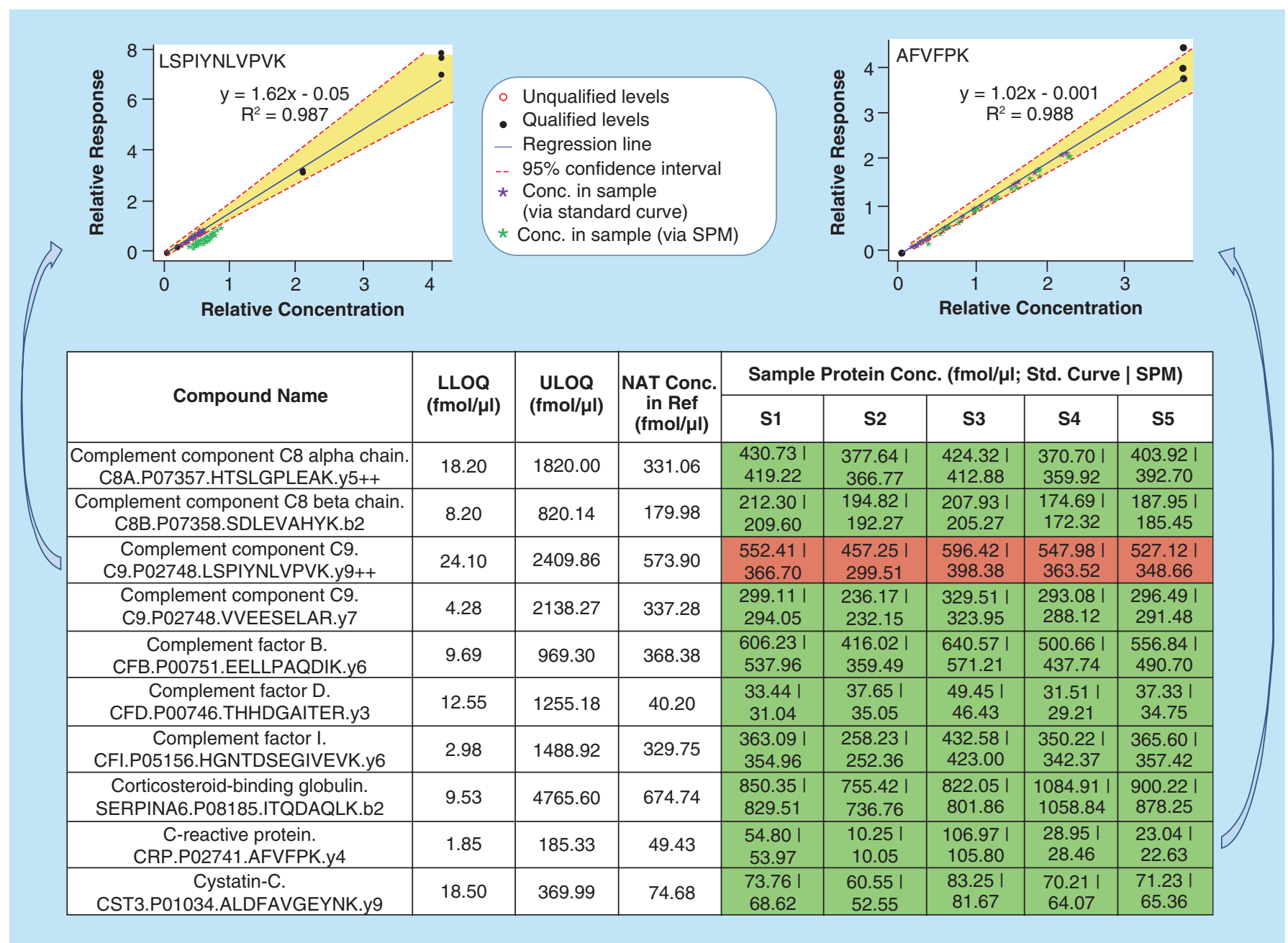

Figure 4. The QA results from Qualis-SIS for a smaller set - 10 peptides (9 proteins) - measured in the sample digests (5 shown for clarity). The insets illustrate an example of acceptable (in green) and unacceptable (in red) values for the 5 sample measurements, where acceptability is defined by its correlation with the sample protein's concentration (derived from regression analysis) and its assay range of linearity, as well as its \% deviation from the SPM ( $20 \%$ threshold in this case). The corresponding input files for Qualis-SIS are provided in Supplementary Tables 4-7. The 'Compound Name' column gives the full protein name, the abbreviated protein name, the UniProt accession number, the peptide, and the product ion monitored, separated by periods.

Nonetheless, it is outside the scope of the present manuscript to investigate the patient results for the purpose of candidate biomarker identification. In such a study, the cohort studied must be carefully selected, and pre-analytical variables (with respect to sample collection, handling, storage, and freeze-thaws) must be harmonized. The resulting data should also be combined with statistical and pathway analyses. After this is done, candidate proteins that are differentially expressed (relative to the control) in 100's of patient samples would qualify for progression to the next phase of the biomarker assessment pipeline - validation where larger sets of samples would be interrogated [44].

Importantly, in the pilot study reported here, the sample protein concentrations were primarily within each assay's range of linearity, which provides confidence in the concentration-balanced SIS mixture and the curve's concentration range (500-fold). If a sample protein concentration is found to lie outside the dynamic range of a given assay or to deviate from the SPM beyond a certain threshold (e.g., 20\%), then the user must decide his own criteria for acceptance. To assist in such assessment, Qualis-SIS includes a quality assessment (QA) page, where the quality of the quantitative results is displayed in a color-coded matrix with the extremes shown in green (for acceptable) and red (for unacceptable) for quick visualization (see Figure 4 for the QA sample results and standard curve examples for a subset of panel targets and patient samples evaluated here).

\section{BAK-76 merits, limitations \& execution tips}

As summarized below, the BAK-76 has several features that make it suitable for the discovery and/or verifi- 
cation of a collection of high-to-moderate abundance, candidate disease protein biomarkers:

i. All target peptides follow conventional selection criteria and have been reproducibly quantified;

ii. Minimum additions of $10 \mu \mathrm{l}$ in sample preparation help improve pipettes accuracy and precision;

iii. SIS peptide mixtures are concentration-balanced for improved analytical precision [27 ];

iv. Narrow MRM detection windows of $1 \mathrm{~min}$ can be utilized since the specified platforms produce high retention-time reproducibility [45];

v. Interference-free peptides are routinely observed on the specified platforms using our indicated gradients and dynamic MRM operation;

vi. No manual integration of the XIC peaks is required during the initial analysis, which allows fast signal verification and the avoidance of the potential subjectivity surrounding peak integration; vii. Protein quantitation is rapid and automated with Qualis-SIS;

viii. Our protein quantitation strategy allows the determination of LOQs, a necessity for establishing the assay limits and verifying the reliability of the determined concentrations.

Despite its merits, there are a few limitations worth mentioning. First, there are no natural (unlabeled) standards (either at the protein or peptide level) supplied to evaluate process efficiency. These would have provided the potential to back-correct for possible losses (toward improved quantitative accuracy) or to quantify the peptides/proteins with alternative techniques (e.g., standard addition [46]). Second, the targets are of moderately high abundance and are designed to be separated under high column pressures (ca. 500 bar) and moderate temperatures (thermostatted at $50^{\circ} \mathrm{C}$ ). An additional drawback is the absence of QC samples (defined as "low", "medium", and "high" based on the measured LOQs [47]) for the

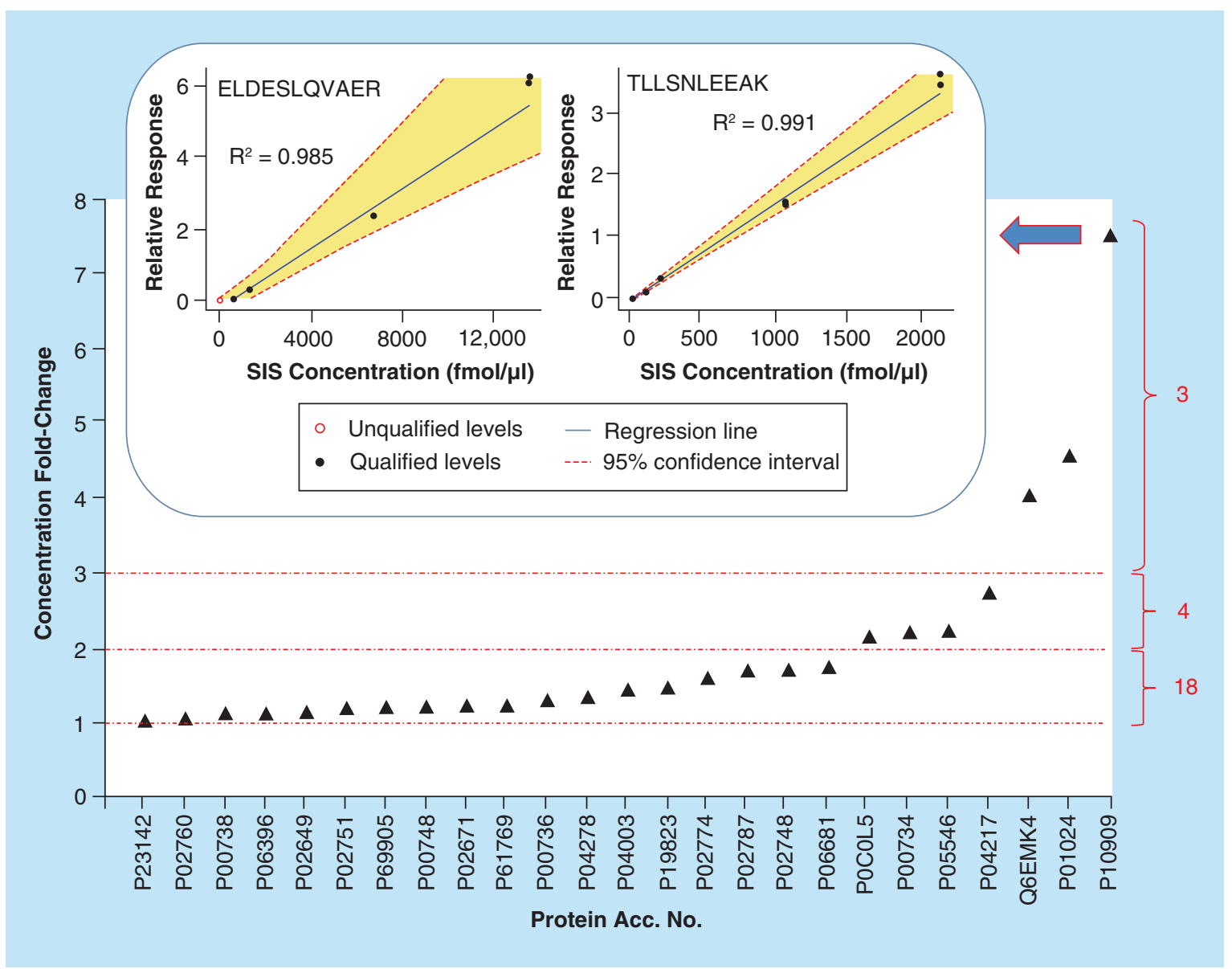

Figure 5. Relative differences in protein concentration for $\mathbf{2 5}$ proteins quantified with $\mathbf{2}$ different proteotypic peptides. Concentrations were obtained from peptide standard curves (as shown in the inset for the control plasma measurements of clusterin (CLU, P10909) using Qualis-SIS. UniProt accession numbers are listed for the proteins, with their associations provided in Supplementary Tables 2 \& 3. 


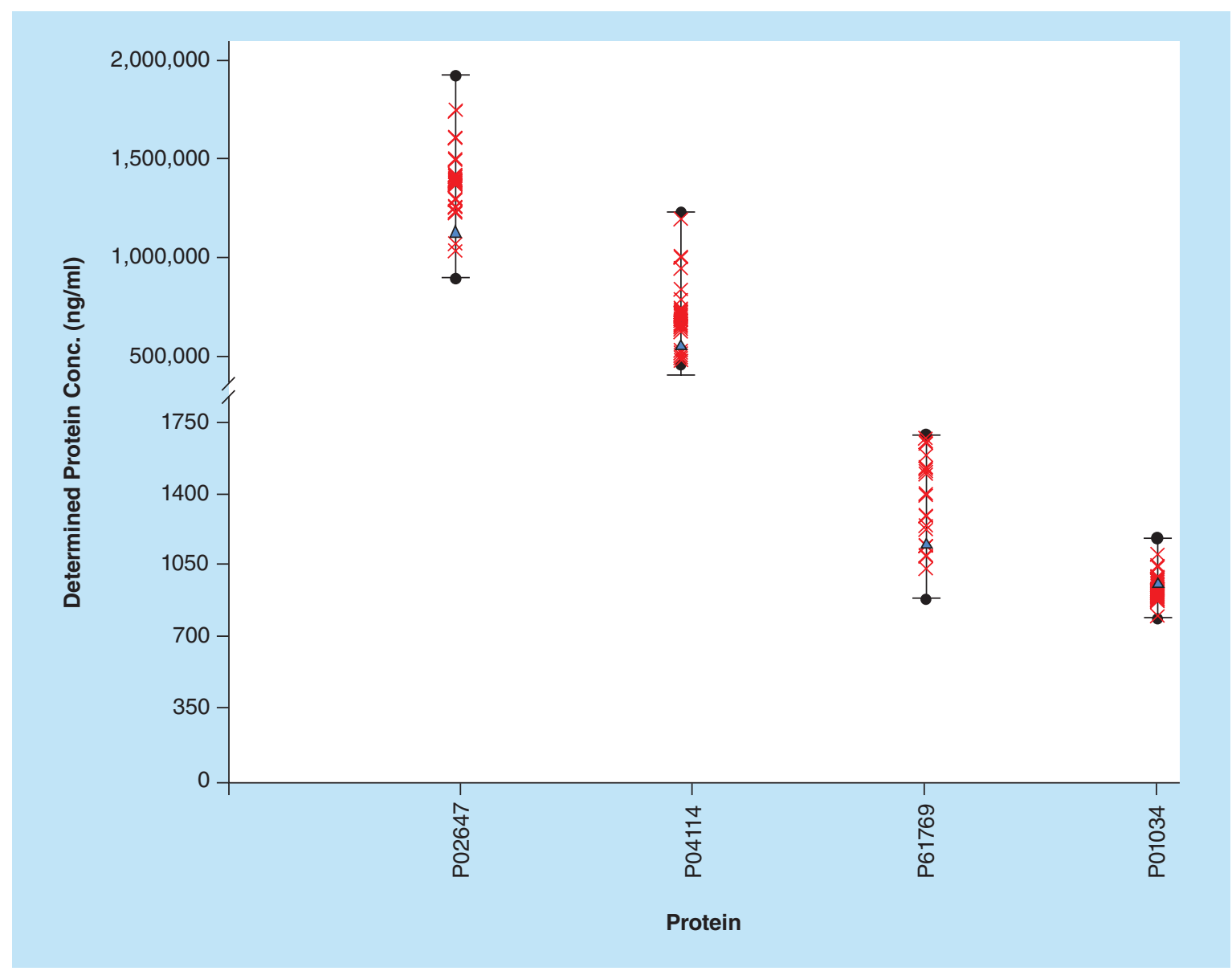

Figure 6. Protein concentrations obtained from MRM analysis of control and patient plasma samples as well as reported immunoassays from clinical measurements. The 2007 clinical reference ranges for 4 proteins (as reported in Supplementary Table 2 from [35]), are indicated as filled black circles, while the control and patient plasma concentrations are shown as filled blue triangles and red crosses, respectively. The reference ranges were determined by immunoassays, while the individual concentrations were determined here by an MRM-with-SISpeptide approach with standard curves.

standard curve. These shortcomings will be addressed in future kits.

To ensure consistent performance while using this kit, it is recommended that the user first standardize his analytical platform and then run periodic checks during the course of the BAK-76 sample analysis. This can be achieved with our daily LC-MS platform performance kit, which requires simple rehydration of the lyophilized peptide mixture before sample processing. Confirmation of acceptable platform performance, as revealed by correlation of the metrics with the reference ranges defined in the QC kit, would provide confidence in the results obtained from the BAK-76.

\section{Reflection \& future perspective}

Over the past decade, the field of targeted proteomics field has advanced in its ability to perform multiplexed MRM experiments ( $>900$ transitions now possible in a single LC-MS run), with isotopically labeled standards (usually peptides) for precise relative protein quantitation. The depth of quantitation (down to low-to-sub ng/ $\mathrm{ml}$ levels) has also improved through the use of up-front enrichment (via anti-protein [48] or anti-peptide [49,50] antibodies) or peptide fractionation (via high $\mathrm{pH}$ followed by low pH LC in an offline [8] or online [51] arrangement). Statistical tools for interpreting the quantitative findings in small-to-moderate sample sets are increasingly being applied for decision-threshold determination (e.g., receiver operating characteristic plots, with performance specificity being determined by the area under the curve). In addition, curated databases of targeted MS assays are now emerging to help researchers perform the assays in their own laboratories (e.g., the cancer-based assay portal through National Cancer Institute's Clinical Proteomic Tumor Analysis Consortium [52]). To aid in the use of these methods by non-LC-MS experts, comprehensive SOPs and sample preparation materials are now being assembled into kits for specific applications. 
In the near future, we anticipate an increasing evaluation of protein biomarkers, and ultimately the clinical adoption of a select few of these as class I-III medical diagnostic assays [53]. This translation is supported by the success of the targeted proteomic approach in small intra-/inter-laboratory studies [54,55-57], its excellent correlation with ELISAs [58] and the presence of triple quadrupole mass spectrometers in the clinic for targeted metabolomic analyses [59]. Further support for the use of MS-based quantitation results from the low cost of the technique and its requirement for small sample volumes (we use $<20 \mu$ l for plasma), as well as its ability to be completely automated (from sample preparation through analysis). In addition, QC standards, which are necessary for routine assessment of instrument/workflow performance, are becoming available for a variety of applications. These standards will be essential for users to quickly verify the performance of their analysis platform before performing the biomarker tests of interest.

Clinical requirements have been described in the US FDA review of a mock pre-submission of a multiplexed MS-based proteomic analysis [60]. With these requirements in mind, we expect method throughput to improve in the coming years, along with improvements in quantitative software facilitating FDA approval. In summary, although few protein biomarkers have been approved in recent years $[53,61]$, we predict that this will change as biomarker assessment methods become standardized and globally implemented for verification and validation studies, in part through the use of kits such as the BAK-76.

\section{Conclusion}

To develop an automated method for standardized protein biomarker assessments in human plasma based on a MRM-with-SIS-peptide approach, we used a workflow that allowed automation of all of the liquid handling steps involved in sample preparation, as well as both automated sample processing and data analysis. This enabled the automated quantitation of 76 plasma proteins in a kit referred to as the BAK-76. The data acquisition/analysis methods and the Qualis-SIS input files, as well as the synthetic peptide standards for the control and patient plasma analysis, are provided, along with the kit's SOP. The targeted plasma proteins are of high-to-moderate abundance, with their derived plasma concentrations spanning $>4$ orders in magnitude, as inferred from their quantitative peptide surrogates. Overall, the use of the BAK-76, together with a QC check of the workflow/platform, will help improve the method reproducibility and transferability between laboratories in biomarker assessment studies; thus, leading to a more rapid and accurate evaluation of these 76 plasma proteins for biomarker discovery and/or verification studies.

\section{Acknowledgements}

The authors are grateful to Reynaldo Interior (Hospital for Sick Children, ON, Canada), Jay Gambee (AAA Service Laboratory, OR, USA), and David Chen (University of British Columbia, BC, Canada) for characterizing our synthetic peptides. Carol E Parker (Editorial Scientist, University of Victoria-Genome BC Proteomics (entre) is recognized for her assistance with manuscript editing.

\section{Financial \& competing interests disclosure}

Genome Canada and Genome British Columbia are thanked for STIC funding and support. C Borchers is the director of the University of Victoria-Genome BC Proteomics Centre and the Chief Scientific Officer of MRM Proteomics, which is responsible for commercializing Centre-related technology developments into PeptiQuant ${ }^{\mathrm{TM}}$ kits for the quantitative proteomics community. The commercialized kits are subsequently distributed through Cambridge Isotope Laboratories. The authors have no other relevant affiliations or financial involvement with any organization or entity with a financial interest in or financial conflict with the subject matter or materials discussed in the manuscript apart from those disclosed.

No writing assistance was utilized in the production of this manuscript.

\section{Supplementary data}

To view the supplementary data that accompany this paper please visit the journal website at: www.future-science.com/ doi/full/10.4155/bio.15.222

\section{Executive summary}

- Protein quantitation kit for assessing the utility of 76 putative disease protein biomarkers.

- Automated workflow encompasses liquid handling sample preparation, LC/MRM-MS sample processing, and quantitative analysis.

- Carefully selected proteotypic ${ }^{13} \mathrm{C} /{ }^{15} \mathrm{~N}$-labeled standard peptides, empirically determined to be free from interferences, help correct for ion suppression, matrix effects, and endogenous peak identification.

- The 101 peptides in the kit are interference-free under the specified conditions and therefore can be automatically integrated before rapid quantitation with our Qualis-SIS software.

- The 76 plasma proteins span >4 orders of magnitude in concentration and are useful for large-scale assessment in biomarker discovery/verification studies. 


\section{References}

Papers of special note have been highlighted as: • of interest; $\bullet$ of considerable interest; $\bullet \bullet$ sources of additional information.

1 Gillette MA, Carr SA. Quantitative analysis of peptides and proteins in biomedicine by targeted mass spectrometry. Nat. Meth. 10, 28-34 (2013).

- Comprehensive review of targeted MS-based methodologies used in quantitative proteomics for biomarker assessment of clinical samples.

2 Picotti P, Aebersold R. Selected reaction monitoringbased proteomics: workflows, potential, pitfalls and future directions. Nat. Meth. 9(6), 555-566 (2012).

-• Seminal overview of MRM-based proteomics with select examples highlighting advances and applications.

3 Boja ES, Rodriguez H. Mass spectrometry-based targeted quantitative proteomics: achieving sensitive and reproducible detection of proteins. Proteomics 12, 1093-1110 (2012).

4 Percy AJ, Chambers AG, Yang J, Hardie DB, Borchers CH. Advances in multiplexed MRM-based protein biomarker quantitation toward clinical utility. Biochim. Biophys. Acta 1844(5), 917-926 (2014).

5 Anderson L, Hunter CL. Quantitative mass spectrometric multiple reaction monitoring assays for major plasma proteins. Mol. Cell. Proteomics 5(4), 573-588 (2006).

6 Percy AJ, Byrns S, Chambers AG, Borchers CH. Targeted quantitation of CVD-linked plasma proteins for biomarker verification and validation. Expert Rev. Proteomics 10(6), 567-578 (2013).

7 Gerszten RE, Asnani A, Carr SA. Status and prospects for discovery and verification of new biomarkers of cardiovascular disease by proteomics. Circ. Res. 109(4), 463-474 (2011).

8 Chambers AG, Percy AJ, Simon R, Borchers CH. MRM for the verification of cancer biomarker proteins: recent applications to human plasma and serum. Expert Rev. Proteomics 11(2), 137-148 (2014).

9 Pan S, Chen R, Brand RE et al. Multiplex targeted proteomic assay for biomarker detection in plasma: a pancreatic cancer biomarker case study. J. Proteome Res. 11(3), 1937-1948 (2012).

10 Reumer A, Maes E, Mertens I et al. Colorectal cancer biomarker discovery and validation using LC-MS/MS-based proteomics in blood: truth or dare?. Expert Rev. Proteomics 11(4), 449-463 (2014).

11 Domanski D, Murphy LC, Borchers CH. Assay development for the determination of phosphorylation stoichiometry using multiple reaction monitoring methods with and without phosphatase treatment: application to breast cancer signaling pathways. Anal. Chem. 82(13), 5610-5620 (2010).

12 Zhao Y, Brasier AR. Applications of selected reaction monitoring (SRM)-mass spectrometry (MS) for quantitative measurement of signaling pathways. Methods 61(3), 313-322 (2013).

13 Omenn GS. The HUPO human plasma proteome project. Proteomics Clin. Appl. 1(8), 769-779 (2007).
14 Ivanov AR, Colangelo CM, Dufresne CP et al. Interlaboratory studies and initiatives developing standards for proteomics. Proteomics 13(6), 904-909 (2013).

15 Percy AJ, Parker CE, Borchers CH. Pre-analytical and analytical variability in absolute quantitative MRM-based plasma proteomic studies. Bioanalysis 5(22), 2837-2856 (2013).

16 Krüger T, Lehmann T, Rhode H. Effect of quality characteristics of single sample preparation steps in the precision and coverage of proteomic studies - a review. Anal. Chim. Acta 776, 1-10 (2013).

17 Wu SL, Choudhary G, Ramström M, Bergquist J, Hancock WS. Evaluation of shotgun sequencing for proteomic analysis of human plasma using HPLC coupled with either ion trap or Fourier transform mass spectrometry. J. Proteome Res. 2(4), 383-393 (2003).

18 Malm J, Fehniger TE, Danmyr P et al. Developments in biobanking workflow standardization providing sample integrity and stability. J. Proteomics 95, 38-45 (2013).

- Standardization guide for biobanking and analysis of human samples in clinical studies.

19 Marko-Varga G, Végvári Á, Welinder C et al. Standardization and utilization of biobank resources in clinical protein science with examples of emerging applications. J. Proteome Res. 11(11), 5124-5134 (2012).

20 Vaught J, Lockhart NC. The evolution of biobanking best practices. Clin. Chim. Acta 413(19-20), 1569-1575 (2012).

21 Escher C, Reiter L, MacLean B et al. Using iRT, a normalized retention time for more targeted measurement of peptides. Proteomics 12(8), 1111-1121 (2012).

- $\quad$ RT prediction tool for targeted proteomic applications across LC systems.

22 Eyers CE, Simpson DM, Wong SC, Beynon RJ, Gaskell SJ. QCAL - a novel standard for assessing instrument conditions for proteome analysis. J. Am. Soc. Mass Spectrom. 19(9), 1275-1280 (2008).

23 Lebert D, Louwagie M, Goetze S et al. DIGESTIF: a universal quality standard for the control of bottom-up proteomics experiments. J. Proteome Res. 14(2), 787-803 (2015).

24 Percy AJ, Chambers AG, Yang J, Borchers CH. Multiplexed MRM-based quantitation of candidate cancer biomarker proteins in undepleted and non-enriched human plasma. Proteomics 13, 2202-2215 (2013).

25 Kuzyk MA, Parker CE, Domanski D, Borchers CH. Development of MRM-based assays for the absolute quantitation of plasma proteins. Methods Mol. Biol. 1023, 53-82 (2013).

26 Percy AJ, Simon R, Chambers AG, Borchers CH. Enhanced sensitivity and multiplexing with 2D LC/ MRM-MS and labeled standards for deeper and more comprehensive protein quantitation. J. Proteomics 106, 113-124 (2014).

27 Kuzyk MA, Smith D, Yang J et al. Multiple reaction monitoring-based, multiplexed, absolute quantitation of 
45 proteins in human plasma. Mol. Cell. Proteomics 8(8), 1860-1877 (2009).

28 Mohammed Y, Percy AJ, Chambers AG, Borchers CH. Qualis-SIS: automated standard curve generation and quality assessment for multiplexed targeted quantitative proteomic experiments with labeled standards. J. Proteome Res. 14(2), 1137-1146 (2015).

- Data analysis software for the rapid and automated quantitation of proteins via linear regression of peptide standard curves or single point measurement.

29 Percy AJ, Chambers AG, Smith DS, Borchers CH. Standardized protocols for quality control of MRM-based plasma proteomic workflow. J. Proteome Res. 12(1), 222-233 (2013).

30 Domanski D, Percy AJ, Yang J et al. MRM-based multiplexed quantitation of 67 putative cardiovascular disease biomarkers in human plasma. Proteomics 12(8), 1222-1243 (2012).

31 Farrah T, Deutsch EW, Omenn GS et al. A high-confidence human plasma proteome reference set with estimated concentrations in PeptideAtlas. Mol. Cell. Proteomics 10(9), M110.006353 (2011)

32 Kiernan UA, Phillips DA, Trenchevska O, Nedelkov D. Quantitative mass spectrometry evaluation of human retinol binding protein 4 and related variants. PLOS ONE 6(3), e17282 (2011).

33 Tu C, Rudnick PA, Martinez MY et al. Depletion of abundant plasma proteins and limitations of plasma proteomics. J. Proteome Res. 9(10), 4982-4991 (2010).

34 Polanski M, Anderson NL. A list of candidate cancer biomarkers for targeted proteomics. Biomarker Insights 2(1), $1-48$ (2007).

35 Hortin GL, Sviridov D, Anderson NL. High-abundance polypeptides of the human plasma proteome comprising the top 4 logs of polypeptide abundance. Clin. Chem. 54, 1608-1616 (2008).

•. Supplementary Table 2 in this paper's Data Supplement contains reference values for protein concentrations from various sources. Current values for these and other diagnostic tests can be obtained from the Mayo Medical Laboratories and the Department of Laboratory Medicine at the National Institutes of Health Clinical Centre at the following websites: www.mayomedicallaboratories. com/test-catalog/appendix/criticalvalues/index.html, and cclnprod.cc.nih.gov/dlm/testguide.nsf/Index?OpenForm, respectively.

36 Josic D, Brown MK, Huang F et al. Proteomic characterization of inter-alpha inhibitor proteins from human plasma. Proteomics 6(9), 2874-2885 (2006).

37 Haab BB, Geierstanger BH, Michailidis G et al. Immunoassay and antibody microarray analysis of the HUPO Plasma Proteome Project reference specimens: systematic variation between sample types and calibration of mass spectrometry data. Proteomics 5(13), 3278-3291 (2005).

38 Anderson L. Candidate-based proteomics in the search for biomarkers of cardiovascular disease. J. Physiol. 563, 23-60 (2005).
39 Griffin JH, Gruber A, Fernández JA. Reevaluation of total, free, and bound protein S and C4b-binding protein levels in plasma anticoagulated with citrate or hirudin. Blood 79(12), 3203-3211 (1992).

40 Argraves WS, Tran H, Burgess WH, Dickerson K. Fibulin is an extracellular matrix and plasma glycoprotein with repeated domain structure. J. Cell Biol. 111(6 Pt 2), 3155-3164 (1990).

41 Agger SA, Marney LC, Hoofnagle AN. Simultaneous quantification of apolipoprotein a-I and apolipoprotein B by liquid-chromatography-multiple- reaction-monitoring mass spectrometry. Clin. Chem. 56(12), 1804-1813 (2010).

42 MacLean B, Tomazela DM, Shulman N et al. Skyline: an open source document editor for creating and analyzing targeted proteomics experiments. Bioinformatics. 26(7), 966-968 (2010).

43 Skyline. Skyline Targeted Proteomics Environment. https://skyline.gs.washington.edu

44 Makawita S, Diamandis EP. The bottleneck in the cancer biomarker pipeline and protein quantification through mass spectrometry-based approaches: current strategies for candidate verification. Clin. Chem. 56, 212-222 (2010).

-. Defines the stages of the protein biomarker pipeline then discusses MRM-based strategies therein for bridging discovery to clinical implementation.

45 Percy AJ, Chambers AG, Yang J, Domanski D, Borchers $\mathrm{CH}$. Comparison of standard-and nano-flow liquid chromatography platforms for MRM-based quantitation of putative plasma biomarker proteins. Anal. Bioanal. Chem. 404(4), 1089-1101 (2012).

46 Keshishian H, Addona T, Burgess $\mathrm{M}$ et al. Quantification of cardiovascular biomarkers in patient plasma by targeted mass spectrometry and stable isotope dilution. Mol. Cell. Proteomics 8, 2339-2349 (2009).

47 US_Food_and_Drug_Administration. Guidance for Industry Bioanalytical Method Validation - September 2013 Biopharmaceuticals (2013). www.fda.gov

48 Wang Q, Chaerkady R, Wu J et al. Mutant proteins as cancer-specific biomarkers. Proc. Natl Acad. Sci. USA 108(6), 2444-2449 (2011).

49 Whiteaker JR, Zhao L, Lin C, Yan P, Wang P, Paulovich AG. Sequential multiplexed analyte quantification using peptide immunoaffinity enrichment coupled to mass spectrometry. Mol. Cell. Proteomics 11(6), M111.015347 (2012).

50 Whiteaker JR, Zhao L, Anderson L, Paulovich AG. An automated and multiplexed method for high throughput peptide immunoaffinity enrichment and multiple reaction monitoring mass spectrometry-based quantification of protein biomarkers. Mol. Cell. Proteomics 9(1), 184-196 (2010).

51 Shi T, Fillmore TL, Sun X et al. Antibody-free, targeted mass-spectrometric approach for quantification of proteins at low picogram per milliliter levels in human plasma/ serum. Proc. Natl Acad. Sci. USA 109(38), 15395-15400 (2012). 
52 Whiteaker JR, Halusa GN, Hoofnagle AN et al. CPTAC assay portal: a repository of targeted proteomic assays. Nat. Meth. 11(7), 703-704 (2014).

53 Füzéry AK, Levin J, Chan MM, Chan DW. Translation of proteomic biomarkers into FDA approved cancer diagnostics: issues and challenges. Clin. Prot. 10(1), 13 (2013).

54 Abbatiello SE, Schilling B, Mani DR et al. Large-scale interlaboratory study to develop, analytically validate and apply highly multiplexed, quantitative peptide assays to measure cancer-relevant proteins in plasma. Mol. Cell. Proteomics 14(9), 2357-2374 (2015).

55 Kuhn E, Whiteaker JR, Mani DR et al. Interlaboratory evaluation of automated, multiplexed peptide immunoaffinity enrichment coupled to multiple reaction monitoring mass spectrometry for quantifying proteins in plasma. Mol. Cell. Proteomics 11(6), M111.013854 (2012).

56 Prakash A, Rezai T, Krastins B et al. Interlaboratory reproducibility of selective reaction monitoring assays using multiple upfront analyte enrichment strategies. J. Proteome Res. 11(8), 3986-3995 (2012).
57 Percy AJ, Tamura-Wells J, Albar JP et al. Inter-laboratory evaluation of instrument platforms and experimental workflows for quantitative accuracy and reproducibility assessment. EuPA Open Proteomics 8, 6-15 (2015).

58 Hoofnagle AN, Becker JO, Oda MN, Cavigiolio G, Mayer P, Vaisar T. Multiple-reaction monitoring-mass spectrometric assays can accurately measure the relative protein abundance in complex mixtures. Clin. Chem. 58(4), 777-778 (2012).

59 Chace DH, Kalas TA. A biochemical perspective on the use of tandem mass spectrometry for newborn screening and clinical testing. Clin. Biochem. 38(4), 296-309 (2005).

60 Regnier FE, Skates SJ, Mesri M et al. Protein-based multiplex assays: mock presubmissions to the U.S. Food and Drug Administration. Clin. Chem. 56(2), 165-171 (2010).

61 Anderson NL. The clinical plasma proteome: a survey of clinical assays for proteins in plasma and serum. Clin. Chem. 56(2), 177-185 (2010). 